\title{
The Edge of a Knife
}

\author{
S Kralj ${ }^{1 *}$ and M Kralj ${ }^{2}$ \\ ${ }^{1}$ Faculty of Natural Sciences and Mathematics, Slovenia \\ ${ }^{2} \breve{S} K U C$, Slovenia
}

*Corresponding author: S Kralj, Faculty of Natural Sciences and Mathematics, Koroška 160, 2000 Maribor, Slovenia

\begin{tabular}{|c|c|}
\hline ARTICLE INFO & ABSTRACT \\
\hline Received: 幽 July 01, 2019 & Citation: S Kralj, M Kralj. The Edge of a Knife. Biomed J Sci \& Tech Res 19(4)-2019. BJSTR. \\
\hline Published: 蔧 July 16, 2019 & MS.ID.003342. \\
\hline
\end{tabular}

\section{Introduction}

Nature is an extremely competitive place. In order to survive living creatures, need to respond fast enough to harmful changes or attacks. For this purpose, natural evolution has armed organisms with various efficient mechanisms enabling sensitive, fast enough and selective responses. One of such relatively simple mechanism is to tune a system close to a relevant phase transition taking place in a medium or a system's organisation. At a phase transition, a system is in general anomalously strongly responsive because the steepness of the corresponding local "energy landscape" is relatively low. Consequently, strong fluctuations in a relevant field describing the system's organisation are possible. For example, a flock of flying birds can be roughly modeled by an Ising-type field, describing their collective orientational order [1]. Analysis of fluctuations in the flock ordering field, which are triggered by a predator attack or some other reasons, reveals that the system is in general just below the phase transition into the disordered phase, in which collective orientational order is absent. Therefore, the proximity of a phase transition arms the system with anomalously strong responsivity using which it could adapt readily to current conditions.

Furthermore, by slightly changing a relevant control parameter one could switch between the competing phases of a phase transition, which exhibit qualitatively different properties. For example, such a mechanism might enable information propagation in nerves according to a "mechanical" nerve theory [2,3]. However, this view contradicts the current mainstream belief, which is based on a Nobel Prize awarded Hodgin-Huxley (HH) model [4]. The latter claims that nerve signals in animals and humans are transmitted electrically and models nerves as electrical objects. However, this model fails to explain the impact of anesthetics on sensitivity of nerves. Namely, various anesthetics trigger the same sequence of effects on increasing their dose in living creatures, including, e.g., flies and humans. On increasing the dose anesthetics first silence memory formation, then pain sensation, consciousness... A wide variety of chemically different anesthetics have the same effect, which cannot be explained within the HH-model.

Furthermore, the electric current following the nerve signal propagation should dissipate heat. However, recently developed extremely sensitive temperature measurement methods indicate that as a nerve pulse propagates it first locally dissipates heat, which is however afterward (within a few thousands of a second) mostly reabsorbed [3]! These phenomena could be explained by treating the nerve propagating signal as a mechanical wave $[2,3]$. This radically different description was proposed already in the mid-1980s [2], and research activities supporting this view have been recently reactivated [3]. The mechanical approach assumes that nerve signals are propagated via mechanical pulses, where an electric signal response is a secondary accompanying effect. To understand key features of the model let us look closer at the axon's outer membrane molecular structure, that is schematically depicted in Figure 1. Its main component is formed by lipid molecules, consisting of a hydrophilic "head" and hydrophobic "tails". The collective structural ordering of lipids could display a phase transition at the critical phase transition temperature $\mathrm{T}_{c}$. For temperatures above $\mathrm{T}_{c}$ the lipids exhibit a liquid phase, where the tails are disordered and strongly fluctuating. Below Tc they condensate in the liquid crystal phase, where the tails are on average aligned along the membrane's surface normal. In the mechanical model, it is assumed that in the normal (nonexcited) state the axon's cell membrane exhibits liquid order. If 
a mechanical pulse is excited, it triggers locally a discontinuous phase transformation into the liquid crystal phase. Namely, a local mechanical deformation acts like a local ordering field, promoting orientational order to lipid tails.

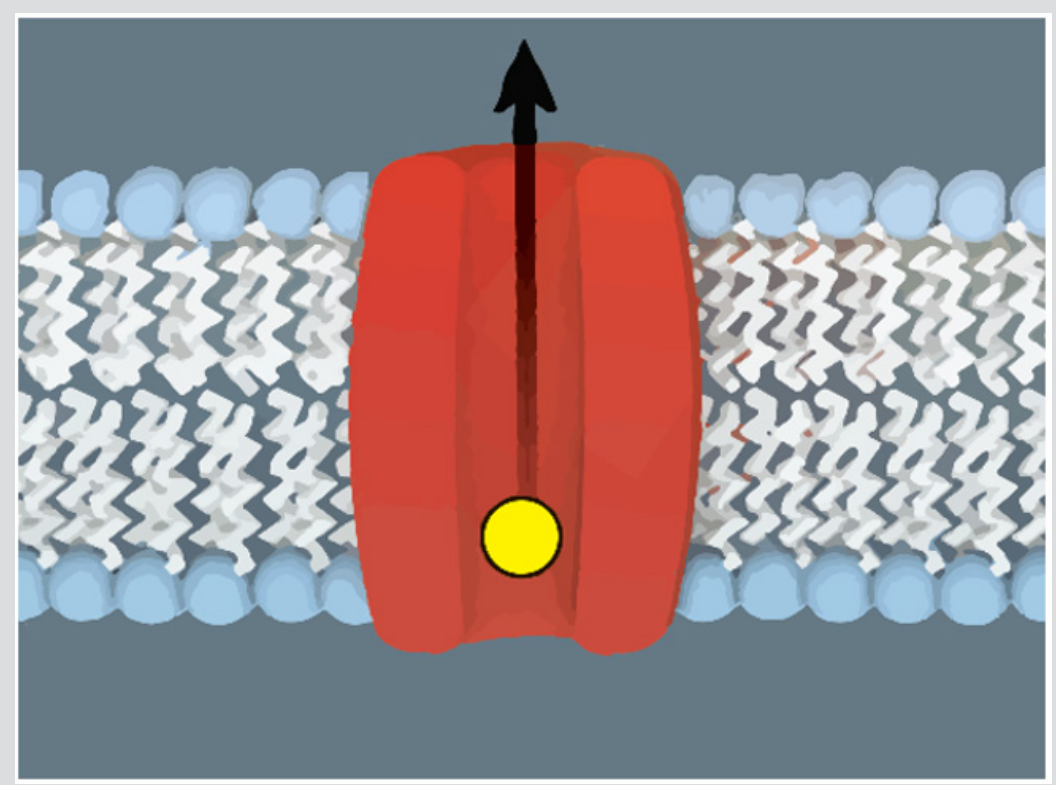

Figure 1: A sketch of a double layer lipid membrane. Lipid molecules possess a hydrophilic (blue colored) "head" and hydrophobic (white colored) "tails". In the liquid phase tails are strongly fluctuating. In the liquid crystal phase, tails are on average orientationally ordered. Membranes, in general, contain several proteins which also strongly influence effective material membrane properties. In the figure, we sketch a (red colored) protein, which can enable transport of (yellow colored) ions through the membrane. Note that its transport properties in general sensitively depend on the surrounding lipid phase state.

In this local phase transition, the latent heat is released, which causes a slight increase in the local temperature. When the propagating deformation exits the region, lipids recover the liquid phase and reabsorb the latent heat, what decreases the temperature. Therefore, the propagating mechanically deformed membrane patch exhibits liquid crystal phase ordering in the surrounding liquid phase. Such behavior explains temperature variations during nerve pulse propagation. Furthermore, it is likely that lipid membranes are piezo electrics, i.e., one could mechanically switch on or off electric voltage [5]. This explains why an electric pulse accompanies the mechanical pulse propagation. Note that in this theory the pulse can propagate due to local liquid crystal phase formation, which provides appropriate conditions for the pulse formation and its propagation. However, if appropriate anesthetics are added, which can enter a lipid membrane, the material properties of the membrane are changed. Consequently, the phase transition temperature can be lowered. If this suppression is large enough, liquid crystal ordering could not be formed and consequently the nerve signal could not propagate. In this case the anesthetic silences the nerve. If this model proves to be correct it might revolutionize our understanding of key biological sensing and information exchange processes.

\section{References}

1. W Bialek, Andrea Cavagnab, Irene Giardinab, Thierry Morad, Edmondo Silvestri, et al. (2012) Statistical mechanics for natural flocks of birds. PNAS 109(13): 4789.

2. I Tasaki, K Kusano, PM Byrne (1989) Rapid mechanical and thermal changes in the garfish olfactory nerve associated with a propagated impulse. Biophys J 55(6): 1033-1040.

3. T Heimburg, DA Jackson (2005) On soliton propagation in biomembranes and nerves. PNAS 102(28): 9790-9795.

4. AL Hodgkin, AF Huxley (1952) A quantitative description of membrane current and its application to conduction and excitation in nerve. J Physiol 117(4): 500-544.

5. Douglas Fox (2018) Brain Cells Communicate with Mechanical Pulses, Not Electric Signals. Scientific American April, 61. 


\section{ISSN: 2574-1241}

DOI: 10.26717/BJSTR.2019.19.003342

S Kralj. Biomed J Sci \& Tech Res

(c) (P) This work is licensed under Creative

Submission Link: https://biomedres.us/submit-manuscript.php

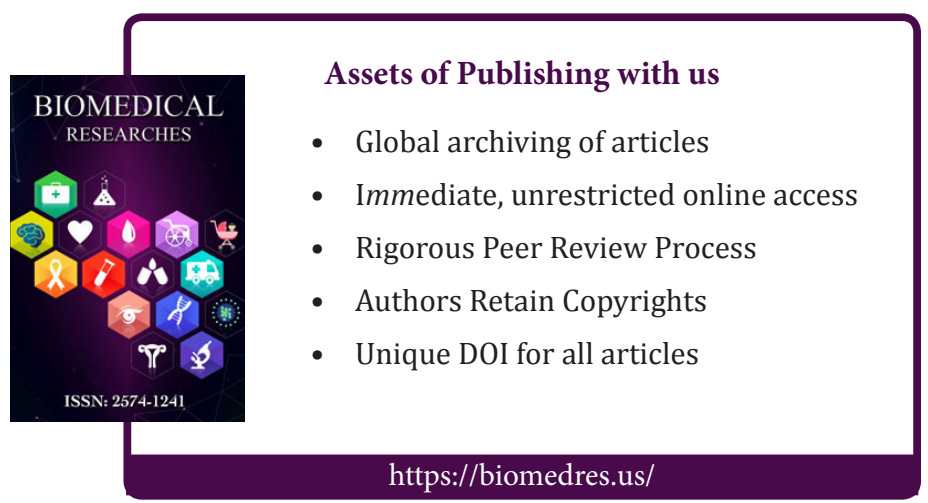

